\title{
Permeation of Computer Technology in Enterprise Management
}

\author{
Huijun Sun \\ Hanjiang Normal University, Shiyan Hubei, 442000, China
}

Keywords: Cloud computing technology, Enterprise management, Cost, Risk, Cloud data, Application.

\begin{abstract}
In the era of informational Internet technology, permeation of computer technology into enterprise management is an inexorable trend. See from the current development, new generation information technology has been listed as one of the seven major emerging industrial strategies, therefore, enterprises also should flexibly use it to control their own development cost and risks. This paper discusses the technical points of cloud computing service mode and related application functions that for enterprise management.
\end{abstract}

\section{Introduction}

Cloud computing mainly involves the value-added services of internet to carry out, a large amount of virtual resources provided by it can bring dynamic extension more possibility to the internet. This computer internet technology was first originated from the research systems of American universities, it is mainly used to reduce universities' research cost and got some achievements. About cloud computing, National Institute of Standards and Technology thought that "It is an internet technological environment at anywhere and use on demand, it shares mechanism of the linkage with internet and computer resources, take use of the minimum service interactions resources to realize the fastest information acquisition and release.”

\section{Analysis on the specific technology of cloud computing service mode in enterprise management}

Cloud computing service mode is favored by the present enterprises mainly because of its comprehensive ability, it can basically meet enterprises' actual management demands in all aspects, and can realize deep integration on the basis of function realization, enlarge enterprises' development advantages. This paper mainly discusses the permeation of computer technology in enterprise management from two aspects of cost and risk.

Enterprise cost management based on cloud computing technology.

The founder of Intel--Gordon.Moore once put forward that semiconductor chip technology follows the traditional Moore's law, he thought that when the commodity price is not changed, the number of transistors contained by semiconductor integrated circuit can be doubled every 18 months, its performance will also be doubled. Cloud computing technology is such a computer information technology that can provide added-value for the system. Set enterprises as example, it has always been devoted into the highly integration of server, switchboard, power hardware and storage device on the aspects of enterprise management and resource integration, sharing and storage, to save resource space for enterprise to control cost. Generally speaking, enterprise cost basing on cloud computing technology mainly covers 3 aspects.

First is "resource pooling", this is a common used resource allocation mechanism for enterprises who use cloud computing technology. Its basic principle is to realize resource allocation and management through the resource manager of enterprise, the through cloud computing to design and integrate resources, then collect these resources into the virtual resource pool for later resource scheduling and using. The support of resource pooling on systematic cloud computing is quite comprehensive, it not only can provide comprehensive information support for enterprise cost management, but also can provide essential resource reference for enterprise hardware and software, save enterprise cost on the development of hardware and software, is an ideal resources integration 
and sharing mechanism.

Second is "demand assignment", it is also the biggest feature of the cloud computing technology. When use cloud computing technology, enterprise must learn to compute added-value cost on demand, thus to lay good foundation for enterprise's benign development of final yield. Demand assignment mainly depends on two technical links of the cloud computing technology: payment system and metering system. Metering system can provide basic frame of resource storage for the cloud computing infrastructure layer, then use service application layer like SaaS to perfect the systematic functions. While the payment system directly provides payment service through connectors of each platform, and the cloud computing technology provides real-time tracking report for payment service, this also provides reliable evidence like payments documents for the real cost of enterprises.

Finally is the virtualization technology, it is also the core technology of cloud computing. In enterprise cost management, it is mainly responsible for the bottom frame of the physical resources to abstractly analyze the operation situation of infrastructures, such as virtual local area network, private network and so on can provide enterprise equipment with virtualized server logic resources supply, realize to operate multiple virtual systems on one physical machine, thus to realize the reuse of physical machine resources. Besides, software virtualization is also a key virtualization technology, it mainly helps enterprise users to complete the development of daily official hardware and software, and it is with higher flexibility, it can realize same effective application on different terminals. Generally speaking, virtualization technology transfers enterprise database resource into dynamic allocated resources, it is very benefit for reducing enterprise purchase and upgrading cost of hardware and software.

\section{Enterprise risk management based on cloud computing technology.}

In order to realize stable development, enterprise have to learn flexibly using scientific management means to reduce and avoid risks, control all kind of uncertain, harmful factors for enterprise development within a bearable range, ensure the stable realization of enterprise' $s$ integrated objective. On the aspect of risk management, cloud computing technology is mainly carries out from 3 aspects of internet safety, backup fault-tolerant and authenticated encryption.

Internet security provides basic guarantee for the internet technology application in modern enterprise, because it can constantly collect virus samples like hacker attack to enrich and update its own rule base, making the application of cloud computing risk management service more smooth and safe. When enterprise' s data flow is treated on several nodes, cloud computing technology can control cloud flow, ensure it will not exceed to the alert peak, and make timely division for the cloud flow. While if enterprise system faces more serious data storm, cloud computing technology will provide system with established software and hardware bi-directional load balancing technology to prevent internet invasion from DDoS form.

On the aspect of backup and fault tolerance, cloud computing technology shows its security and stability. Especially in the current enterprise system with more and more data, there are more and more business needs, treatment links also become more and more complicated, thus the error incidence of cloud system will also increases. At this time, backup and fault tolerance will be of help, this technology operates the hardware and software in the system, such as router, power supply, middleware, through multiple nodes distribution, to increase a certain amount of data copy quantity for cloud computing, strengthen the backup function of the system, to make copy quantity always keeps a certain amount. Moreover, the fault- tolerance performance of managed node based on Worker can be improved under this technology, when node Worker and managed node Broker go wrong, it can help system timely switch to corresponding right copy position and let copy to complete the systematic work task within the plan.

Finally is the authenticated encryption, cloud computing is with feature of sharing storage data, it realize important development of important data through key management, then based on user identity authentication and the sharing resources in the resources pool to combine to design, generate functional service layer such as SaaS、PaaS for the system, making cloud computing divides into 
public and private cloud computing, two kinds of internet management environment, effectively control user access. Under these two kinds of environment, all kinds of operation standards are compatible with each other, and they also realized strengthen on the user authentication mechanism, its strain capacity for enterprise's all kinds of role designer and technical requirements is considerable $^{[1]}$.

\section{Permeation of cloud computing service application in enterprise management}

Enterprise management takes use of the permeation of cloud computing service application technology to realize technology research, product production and marketing orientation, meeting market requirements is rather necessary, it also has an improvement on enterprises sustainable development mechanism. This paper specifically discusses on the technology permeation application process of virtualized cloud computing desktop and cloud data central service in enterprise management.

\section{Application of cloud desktop in enterprise management.}

1) Technology introduction

Cloud desktop is one kind of the cloud computing virtualization technology, it can directly operate cloud system, and connect users' terminal equipment internet at any time any where, it also can be called as remote desktop access system. Cloud desktop mainly involves virtualization technology and is installed and allocated with corresponding virtual machine system, this system can provide enterprise management with needed computing and storage and other hardware service, it also can improve utilization efficiency through data sharing to improve utilization efficiency for the system. Specifically speaking, the lay out of virtual cloud desktop mainly involves 3 parts: data center, user area and network connection area.

Data center is the core area of the resource layout of this cloud desktop, operation and maintenance staffs operate all the enterprise management technology layout in this area. It includes the concentrated storage of data, the integration and sharing of CPU, storage resources and the safety protection on data; user area is mainly responsible to connect users' terminal equipment to realize the usage of cloud desktop; while the internet area is the basic area of user area and the data center, it provides high-speed internet connection service, to realize the environment for internet information needed by the cloud desktop, it is the power source of all kinds of cloud application service.

\section{2) Application process}

Cloud desktop is based on virtualization technology to realize function frame design and application, its terminal takes use of VDM virtual desktop management system and OPV data center virtual system.

It technology application process is that first open the remote terminal service, then through VCN module to connect system manager. Manager will operate secure authentication and authorization for system users and supports to use LDAP. After authentication, connection manager will open switch-on countermeasures to OPV-Manager, receive the application requirement from users' virtual machine, check application users' virtualization machine state, open the virtual machine after conforming standards to make data treatment and analysis. At last, OPV-Manager will feedback data treated by virtual machine to the connection manager, at this time, users can directly realize actual access to the virtual machine through connection manager.

Compared with the terminal of traditional PC frame, cloud desktop is more safe and reliable, because it accesses to the computer server through virtual desktop, actually, the data did not be stored in the client terminal, in addition to the security isolation function of internal and external network, therefore, server's data backup protection can be stable, and the speed of backup recovery is also improved.

On the other hand, cloud desktop also will allocate relative lower terminal to bring greater cost saving, because its maintenance and treatment are automatic, therefore, during the daily application process, its requirements on operation and maintenance are also pretty low, it saved lots of human 
resource and material cost. At last, on the aspect of resource utilization, because of the concentrated layout of the cloud desktop, customers are more flexible on using server resources, it cleans the normal environment of desktop computing resource, and make resources be allocated more reasonable. According to statistics, the server resource utilization rate under the cloud desktop is $60 \%$ higher than traditional desktop system or ever more ${ }^{[2]}$.

Application of cloud data center in enterprise management.

OPV-Manager of the cloud data center is responsible for the management of virtual machine in enterprise system, realize the unified assignment of resources and timely supervise on the system operation environment. Specifically speaking, firstly, it realized the management of virtual life period, OPV-Manager provides complete life period management for enterprise management system virtual machine, it covers functions from initial establishment, middle operation, suspension, mirror image, clone to reallocation and deletion. While on the aspect of user management, OPV-Manager can provide technical support for local user management, and involves LDAD and Webseal components to realize the third party identity authentication and cross-domain identity authentication. On the aspect of dynamic resource management, OPV-Manager will establish virtual resource pool through various physical hardware resources like CPU, network card and so on, and design multiple sharing mechanism for resource pool, complete application on virtual machine under the dynamic and intelligent allocating strategy, final to realize the fully allocation and reduced function of computer resources.

The most important are security management and IP service, OPV-Mananger provides enterprise system with stratified safe service, it mainly supports the mutual control, access and address switching among virtual machines, also matches with the security strategy of the application allocation, supervise users' system logging in operation and resource application. After users' system data being with encryption, even the original documents lost, it will not cause big data leakage; on the contrary, on the aspect of IP service, IP service provided by OPV-Manager is diversified, such as functions like DNS, DHCP, PXE, NAT, all of them are components of IP service management. IP service can reach the same function features with virtual machine, can realize independent operation on any physical nodes. If IP service breaks down, it can transfer data to the neighbor server for constant treatment and operation, this provides an effective guarantee for the sustainable and safe operation of the system ${ }^{[3]}$.

\section{Conclusion}

To sum up, the permeation and application of cloud computing technology in enterprise management is rather flexible, safe and reliable, it gives full play of all the functional features of the computer system, and can combine enterprise' real needs to explore more resource and relevant service model in enterprise management, guarantees enterprise's stability on cost and risk management.

\section{References}

[1] Yong Feng. Analysis and Application of Cloud Computing Service Model for Enterprise Management, Tianjin University, 2014.

[2] Wu Yong. Application of Computer Technology in Enterprise Management, Research on Urban Construction Theory (Electronic Edition), 2015(22):2309-2309.

[3] Wang Kaiyi. Brief Analysis on the Application of Computer Technology in Enterprise Management, Digital Technology and Application, 2014(2):113-113. 